Published in final edited form as:

Nano Lett. 2007 ; 7: 3854-3858.

\title{
Identification of DNA base-pairing via tunnel-current decay
}

\author{
Jin $\mathrm{He}^{\dagger}$, Lisha Lin ${ }^{\dagger} \ddagger$, Peiming Zhang ${ }^{\dagger}$, and Stuart Lindsay ${ }^{\dagger}, \ddagger, \S,{ }^{*}$ \\ $\uparrow$ Biodesign Institute, Arizona State University, Tempe, AZ 85287, USA \\ $\$$ Department of Chemistry and Biochemistry, Arizona State University, Tempe, AZ 85287, USA \\ § Department of Physics, Arizona State University, Tempe, AZ 85287, USA
}

\begin{abstract}
We propose a new approach for reading the sequence of a DNA molecule passing between electrodes on a nanopore, using hydrogen-bond mediated tunneling signals. The base-electrode interaction is modeled using a nucleobase functionalized STM probe that is pulled away from a nucleoside monolayer. Watson-Crick recognition results in slow-decay of the tunnel current, uniquely characteristic of the base-pair in over half the reads. Thirteen independent reads would yield the desired $99.99 \%$ accuracy.
\end{abstract}

\section{Keywords}

DNA sequencing; single molecule recognition; hydrogen bond mediated tunneling

Radical new approaches are required to bring down the $\operatorname{cost}^{1}$ and increase the read lengths ${ }^{2}$ of DNA sequencing. A nanopore is a nanofluidic device that separates two liquid compartments, and contains a small hole that connects the two electrolyte reservoirs. The hole is so small that DNA passes through one base at a time. This paper describes one component of a readout system that may be attached to a nanopore, reading the identities of the bases as they pass the pore.

DNA bases can be identified statistically in nanopore translocation events, but the distinct single-molecule signals essential for sequencing are not yet available. ${ }^{3}$ One intriguing proposal is to use electron tunneling to identify bases electronically as they transit the nanopore, ${ }^{4-6}$ though there is some debate about the feasibility of this approach. ${ }^{7}$ Ohshiro and Umezawa ${ }^{8}$ have shown that hydrogen-bond (H-bond) mediated interactions between DNA bases can be 'imaged' in a scanning tunneling microscope (STM) using a tip functionalized with one of the four DNA bases. However STM imaging of DNA is challenging, even in ultrahigh vacuum, 9 and interpretation of STM contrast can be problematic. ${ }^{10}$ We propose combining nanoporetunneling with hydrogen-bond mediated molecular recognition in a scheme we call Sequencing by Recognition. Each base is identified by a characteristic current-distance response as single stranded DNA passes by chemically-functionalized electrodes in a nanopore. In this scheme, four separate reading heads would be used, each one responsible for identifying one of the four DNA bases. The circuit would be completed by a second electrode funtionalized with a reagent that hydrogen bonds the phosphates on the opposite side of the DNA. Here, we demonstrate the feasibility of electrically reading DNA base pairing, showing that with a guaninefunctionalized STM probe, measurements of the decay of tunnel current with distance clearly distinguish Watson-Crick G-C pairs from G-T 'wobble' base-pairs. The integrated current signal identifies G-C interactions unambiguously in over half the single molecule 
measurements made in an organic solvent. Surprisingly, the signals are nearly as distinctive in water, though the transport mechanism appears to be different. We have not detected any false positives in the readout, so the accuracy is limited only by events in which identification fails. These failures occur in less than half the reads, so thirteen independent reads would yield the desired $99.99 \%$ accuracy $\left(0.513<10^{-4}\right) .11$

We have studied tunneling in monolayers of deoxycytidine and thymidine attached to $\mathrm{Au}(111)$ using gold STM tips functionalized with 8-mercaptoguanine. Thiophenol-, 6-

mercaptocytosine-functionalized and unfunctionalized tips were used as controls. We chose thiolated nucleosides to overcome the tendency of bases to lie flat on gold. ${ }^{12} 5^{\prime}$-thiodeoxycytidine ( $\mathrm{i}$ - referred to as "C") and 5'-thio-thymidine (ii - referred to as " $\mathrm{T}$ ") were synthesized following published protocols. ${ }^{13-15}$ They form Watson-Crick (i) and G-T wobble (ii) base pairs with guanine: 16
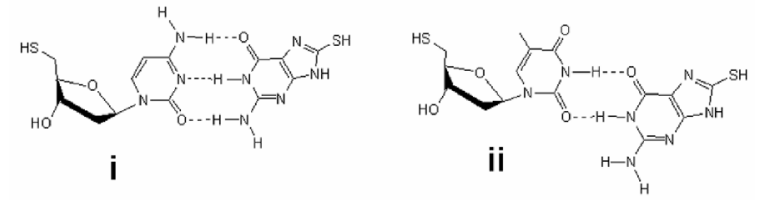

STM images (supporting Information) show a two-fold increase in contrast when a surface containing cytosines is imaged with an 8-mercaptoguanine functionalized STM probe, confirming the phenomenom reported by Ohshiro and Umezawa 8 and extending their result to these nucleosides. Striking though this effect is, an image-based analysis presents several challenges: (a) It is not obvious how to choose the low-point in the intensity distributions. (b) Topography and electronic effects are convoluted in the images. (c) Without a first-principles simulation of the images, we cannot even be sure that 'spots' in the image correspond to nucleotide positions. (c) Long DNA molecules lying on a surface are unlikely to be free from secondary structure which obscures access to bases. In order to better understand the origin of the contrast modulation in the STM images, we measured the decay of tunnel current with distance (i-z curves) over various modified and unmodified surfaces. I-z curves have the advantage of being normalized, starting at approximately the same height above the surface each time, but they are notoriously sensitive to fluctuations in geometry and contamination.

Remarkably repeatable current-distance curves were obtained in an organic solvent, as illustrated in Figure 1. This shows i-z data taken in trichlorobenzene with bare tips (green curves), thio-phenol modified tips (orange curves) and 8-mercaptoguanine modified tips (black curves) on bare gold (a), a 5'-thio-thymidine SAM (c) and a 5'-thio-deoxycytidine SAM (d). Data are also shown for a mercaptocytosine functionalized probe with a 5'-thio-thymidine SAM (b). The plots show an overlay of all the data collected in a typical run in which the probe was successfully functionalized (>60\% of the probes - see supporting information). It is immediately obvious from the raw data that there is large difference between $\mathrm{H}$-bond mediated curves and non H-bond mediated curves (Fig. 1a vs. Figs. 1b, c, d). Furthermore, the two-Hbond G-T wobble base-pair (Fig. 1c) gives a signal that is quite distinct from the three-H-bond G-C Watson Crick base-pair (Fig. 1d). C and T form less stable hydrogen bonded structures ${ }^{17}$ and curves taken with a C-tip on a T-substrate lie between the controls and the GT signals (blue lines, Fig. 1b).

One reason for the remarkable robustness of these measurements may lie with the bonded nature of the junctions. The tunneling path is composed of chemically-bonded components from one metal surface to the other, a prerequisite for repeatable single-molecule electronic measurements. ${ }^{18}$ 
Do these curves probe intrinsic electronic differences between G-C and G-T hydrogen bonds, or rather the mechanical strength of the various types of bonds? To further characterize the interaction, we fitted the curves with two exponentials,

$$
\begin{gathered}
i=i_{0} \exp -\beta_{1} z 0<z<z_{c} \\
i=i\left(z_{c}\right) \exp -\beta_{2}\left(z-z_{c}\right) z_{c}<z .
\end{gathered}
$$

In order to illustrate the fitting process, we show examples of fits to a few typical curves in Figure 2. The decay consists of two regions. The current initially decays slowly (regions labeled $\beta_{1}$ in Fig. 2) and then more rapidly thereafter (regions labeled $\beta_{2}$ in Fig. 2). Fits to equations $1 \mathrm{a}$ and $1 \mathrm{~b}$ yield values for $\beta_{1}, \beta_{2}$ and $z_{c}$. Average values for the parameters $\beta_{1}$ and $\beta_{2}$ are listed in Table 1 (histograms of all the data are given in the supporting information). Values of $\beta$ are similar to the decay constants in the control experiments, so we conclude that this region corresponds to tunnel current decay in the solvent, the H-Bonds having broken. Values of $\beta$ are much smaller than either those of $\beta$ or the control data. Values of $\beta$ for G-C and G-T basepairs are quite similar to each other (but G-T base-pairs appear to form less frequently supporting information). The main difference between G-C and G-T base-pairs lies in the extent of the curves, as quantified by the break-point $z_{c}$. Histograms of this quantity are shown in Figures 3 a-c. G-C basepairs give signals that remain large out to ca. $1 \mathrm{~nm}\left(0.5 \mathrm{~nm}<z_{c}<1.25\right.$ $\mathrm{nm})$. G-T basepairs extend to ca. $0.5 \mathrm{~nm}\left(0.25 \mathrm{~nm}<z_{c}<0.75 \mathrm{~nm}\right)$. The $\mathrm{G}$ controls on bare surfaces show some evidence of a non-specific interaction (Figure 3a) but the extent is small. Thus, we conclude that dominant effect lies with how far the base-pairs can be pulled before the H-bonds are broken. This analysis is limited in scope, because $20 \%$ of the curves failed to show a distinctive break, so we calculated the charge transferred in each pull by integrating the raw current vs. time data, a procedure that can be applied to all the data collected (Figures $3 \mathrm{~d}-\mathrm{f}$ ). We have also included data for a SAM made with an equimolar C/T mix (Figure 3g). It is immediately obvious that G-C interactions are uniquely identified by charge transfers of 15 to $20 \mathrm{pC}$ in these tunneling conditions (set-point conductance $=6 \mathrm{nS}$, withdrawal speed $=$ $133 \mathrm{~nm} / \mathrm{s}$ ). Some of the G-C charge transfers overlap the G-T data, possibly because of non Watson-Crick G-C basepairs or strained configurations owing to the rigid attachment of the 8mercaptoguanine to the tip, but these artifacts do not provide false positive reads for G-C basepairs.

These data were obtained in trichlorobenzene primarily because we expected that hydrogen bonding would be stronger in an organic solvent than in water. We repeated these measurements in an aqueous environment using polyethylene-insulated probes, and the raw data are displayed in Figure 4. The i-z curves in water clearly distinguish Watson-Crick vs. wobble basepairing also (Figs. $4 \mathrm{c}$ and $4 \mathrm{~d}$ ). The signals from C-T (Fig. 4b) are much more spread out (Fig. 4c) in this case, consistent with the known role of water bridges in C-T bonding. 17 The charge transfer signals obtained in water (Figures $5 \mathrm{a}-\mathrm{c}$ ) are not very different from those obtained in tricholorobenzene. G-C basepairs are characterized (uniquely for $50 \%$ of the data) by a charge transfer of $>10 \mathrm{pC}$, while G-T and some G-C signals lie in the 5 to $10 \mathrm{pC}$ range. These data are at odds with our expectation that basepairs should rupture more readily in water. However, a side-by-side comparison of the i-z curves in trichlorobenzene and water (Figures $5 \mathrm{~d}-\mathrm{g}$ ) shows that the curves obtained in water are quite different in shape from those obtained in trichlorobenzene. They have a complex structure (Figs. 5 e and g) while curves obtained in tricholorobenzene (Figs. $5 \mathrm{~d}$ and f) are almost all well-fitted by equations (1a) and (1b). We speculate that the basepairs do indeed rupture sooner in water, but that signals are sustained by water bridges between the bases.

The decay constants in the first region of all of these experiments $\left(\beta_{1}\right)$ are much smaller than in solvent $\left(\beta_{2}\right)$ and too small to be interpreted as true tunnel current decay constants.19 One 
interpretation is that the distortion of the H-bond is much smaller than the overall extension of the junction because the H-bonds are stronger than other components of the gap. $20 \mathrm{We}$ model the tunnel junction as composed of two elastic elements in series, one a spring of constant $\kappa_{1}$ representing the various bonds (other than H-bonds) and the deformable probe2 1 and the second corresponding to the hydrogen bonds themselves, with spring constant $\kappa_{H}$ and separation $x_{H}$. Consequently, $x_{H}$ is less than $x_{T}$ by a factor $\frac{\kappa_{1}}{\kappa_{1}+\kappa_{H}}$. Thus the apparent decay constant, $\beta_{a p p}$ can be stated in terms of the real electronic decay constant, $\beta$, as

$$
\beta_{a p p}=\beta \frac{\kappa_{1}}{\kappa_{1}+\kappa_{H}} .
$$

The experimental curves are reproduced using reasonable values for $\beta$ if the hydrogen bonds are a few times stiffer than other bonds in the tunnel junction (supporting information).

In conclusion, we have shown that an electrical signal uniquely characteristic of Watson-Crick base-pairing can be obtained in about half the tunneling measurements made by pulling apart G-C or G-T base-pairs. Because this scheme does not appear to produce false positives, it should only be necessary to oversample to the extent required to obtain a positive signal at least $99.99 \%$ of the time (the NIH target accuracy for sequence data). This could be achieved with just thirteen independent reads for a G-functionalized reading head, assuming that sequence data were accurately aligned in each run. Clearly modified bases (such as 8mercapto-2-aminoadenine) would be required for identification of the A-T basepair and mismatches may be more of a problem in an aqueous environment. With these caveats, our results suggest that single molecule sequencing may be feasible using H-bond mediated tunneling as a sensor in a nanopore reader.

\section{Supplementary Material}

Refer to Web version on PubMed Central for supplementary material.

\section{Acknowledgements}

We acknowledge useful discussions with Otto Sankey, Iris Visoly and Henry White. This work was supported by grants from the Biodesign Institute, Arizona Technology Enterprises and the DNA sequencing technology program of the NIH (1 R21 HG004378-01)

\section{References}

1. Fredlake CP, Hert DG, Mardis ER, Barron AE. What is the future of electrophoresis in large-scale genomic sequencing? Electrophoresis 2006;27:3689-3702. [PubMed: 17031784]

2. Chan EY. Advances in sequencing technology. Mutation Research-Fundamental and Molecular Mechanisms of Mutagenesis 2005;573:13-40. [PubMed: 15829235]

3. Zwolak M, Di Ventra M. Physical approaches to DNA sequencing and detection. Reviews of Modern Physics. 2007in press, available at arXIV: 0708.2724

4. Lee, JW.; Thundat, T. DNA and RNA sequencing by nanoscale reading through programmable electrophoresis and nanoelectrode-gated tunneling and dielectric detection 6,905,586; June 14, 2005; 2005.

5. Zwolak M, Di Ventra M. Electronic Signature of DNA Nucleotides via Transverse Transport. Nano Lett 2005;5:421-424. [PubMed: 15755087]

6. Lagerqvist J, Zwolak M, Di Ventra M. Influence of the Environment and Probes on Rapid DNA Sequencing via Transverse Electronic Transport. Biophys J 2007;93:2384-2390. [PubMed: 17526560]

7. Zhang XG, Krstic PS, Zikic R, Wells JC, Fuentes-Cabrera M. First-Principles Transversal DNA Conductance Deconstructed. Biophys J 2006;91:L04-L06. [PubMed: 16679371] 
8. Ohshiro T, Umezawa Y. Complementary base-pair-facilitated electron tunneling for electrically pinpointing complementary nucleobases. Proc Nat Acad Sci 2006;103:10-14. [PubMed: 16373509]

9. Shapir E, Cohen H, Borovok N, Kotlyar AB, Porath D. High-Resolution STM Imaging of Novel Poly (G)-Poly(C) DNA Molecules. J Phys Chem B 2006;110:4430-4433. [PubMed: 16509745]

10. Clemmer CR, Beebe TP. Graphite: A mimic for DNA and other Polymers. Science 1991;251:640 642. [PubMed: 1992517]

11. Lander ES, et al. Initial sequencing and analysis of the human genome. Nature 2001;409:860-921. [PubMed: 11237011]

12. Tao NJ, DeRose JA, Lindsay SM. Self Assembly of Molecular Superstructures studied by in situ STM: The DNA bases on Au(111). J Phys Chem 1993;97:910-919.

13. Henningfeld KA, Arsian T, Hecht SM. Alteration of DNA primary structure by DNA topoisomerase I. Isolation of the covalent topoisoneraseI - DNA binary complex in enzymatically competent form. J Am Chem Soc 1996;118:11701-11713.

14. Yelm KE. A Simple Method for in situ Generation of Thiols from Thioacetates. Tetrahedron Letters 1999;40:1101-1102.

15. Zheng TC, Burkart M, Richardson DE. A General and Mild Synthesis of Thioesters and Thiols from Halides. Tetrahedron Letters 1999;40:603-606.

16. Early TA, Olmsted J, Kearns DR. Base pairing structure in the poly d(G-T) double helix: wobble base-pairs. Nucleic Acid Research 1978;5:1955-1970.

17. Allawi HT, SantaLucia J. Thermodynamics of internal C.T mismatches in DNA. Nucleic Acid Research 1998;26:2694-2701.

18. Cui XD, Primak A, Zarate X, Tomfohr J, Sankey OF, Moore AL, Moore TA, Gust DGH, Lindsay SM. Reproducible measurement of single-molecule conductivity. Science 2001;294:571-574. [PubMed: 11641492]

19. Vaught A, Jing TW, Lindsay SM. Non-exponential tunneling in water near an electrode. Chemical Physics Letters 1995;236:306-310.

20. Lindsay SM, Thundat T, Nagahara LA. Adsorbate deformation as a contrast mechanism in STM images of biopolymers in an aqueous environment: Images of the unstained hydrated DNA double helix. J Microscopy 1988;152(Pt 1):213-220.

21. Xu B, Xiao X, Tao NJ. Measurements of Single-Molecule Electromechanical Properties. J Am Chem Soc 2003;125:16164-16165. [PubMed: 14692738] 

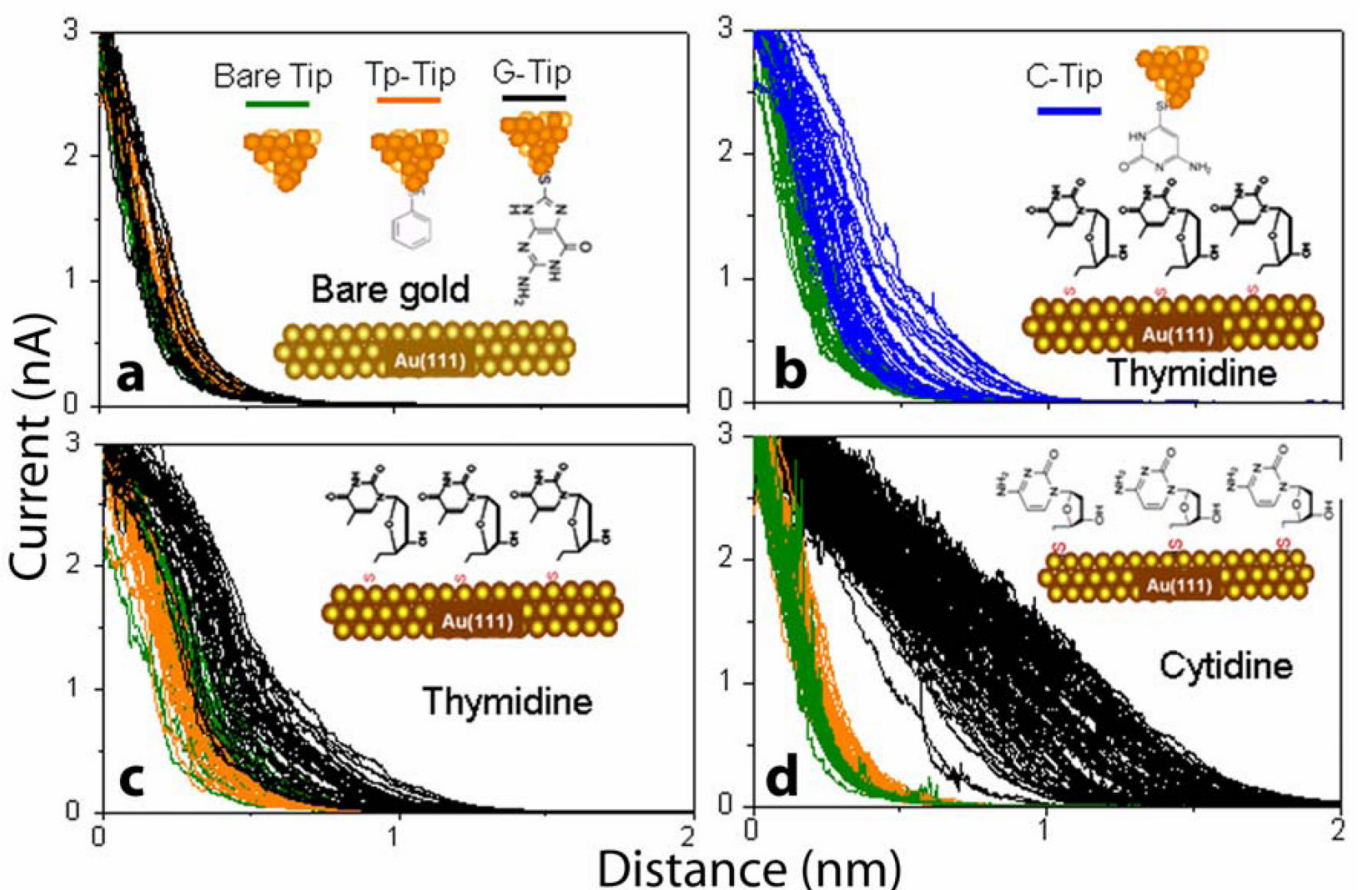

Figure 1.

Raw current vs. distance data for a bare Au tip (green), thiophenol (Tp) -modified tip (orange) and G-modified tip (black) on (a) Bare gold, (c) a thymidine SAM and (d) a deoxycytidine SAM. Data taken with a C-funtionalized probe on a thymidine SAM are shown in (b) (blue lines, green lines are data for the bare probe). Initial conditions are $\mathrm{i}=3 \mathrm{nA}, \mathrm{V}=0.5 \mathrm{~V}$, retraction speed $=133 \mathrm{~nm} / \mathrm{s}$. Data were taken in trichlorobenzene. 


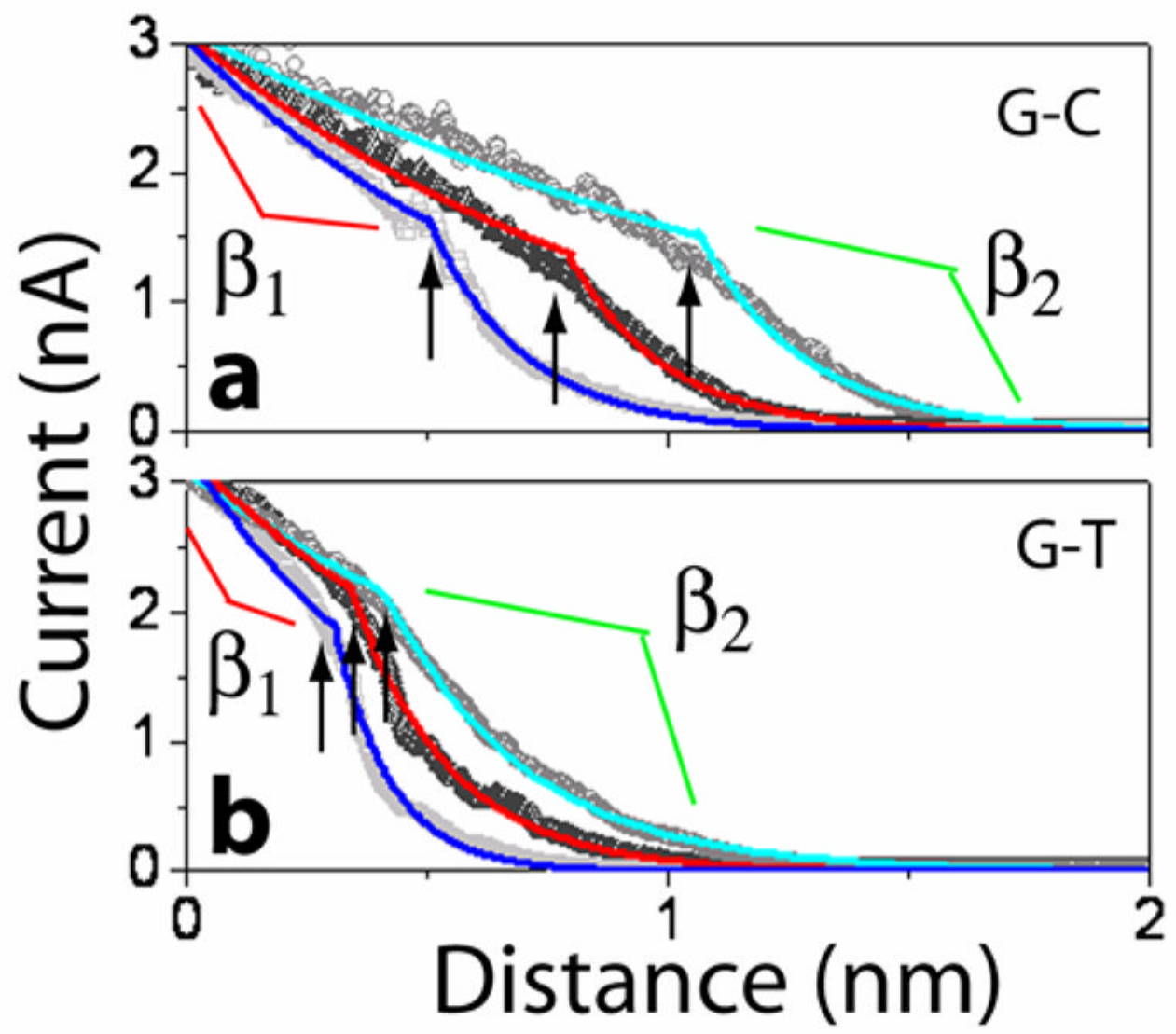

Figure 2.

Some typical curves for a $G$ probe and a deoxycytidine surface (a) or a thymidine surface (b) showing fits to equations $1 \mathrm{a}$ and $1 \mathrm{~b}$. Arrows point to the transition between the slow decay $\left(\beta_{1}\right)$ and the rapid decay $\left(\beta_{2}\right)$ regions at a distance $z_{c}$. 


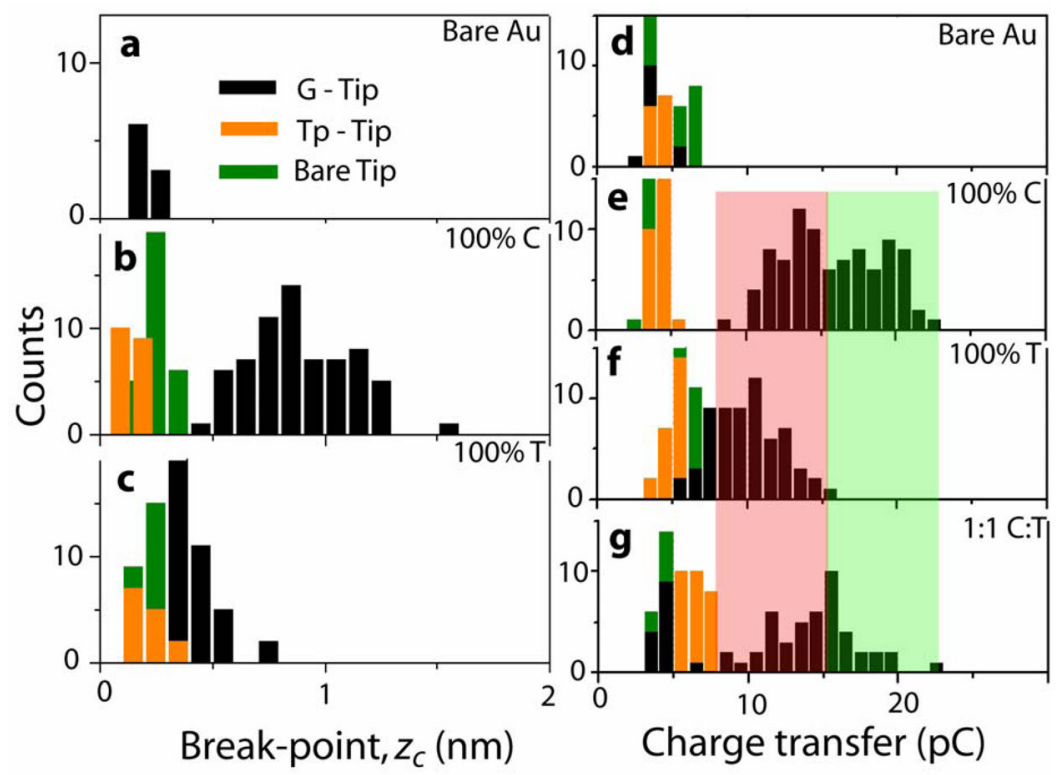

Figure 3.

a-c Histograms (black bars) of the values of the breakpoint, $z_{c}$, for bare Au (a), a deoxycytidine SAM (b) and a thymidine SAM (c) for some of the i-z curves shown in Figure 1. Panels d-g show histograms of the charge transferred in each retraction (all data) for (d) bare gold, (e) a deoxycytidine SAM, (f) a thymidine SAM and (g) a SAM made with an equimolar mix of C and T. Black bars are for an 8-mercaptoguanine functionalized tip, orange bars are for a thiophenol functionalized tip and green bars are for a bare tip. The green shaded block indicates the region of unambiguous signals for $\mathrm{G}-\mathrm{C}$ basepairs. 


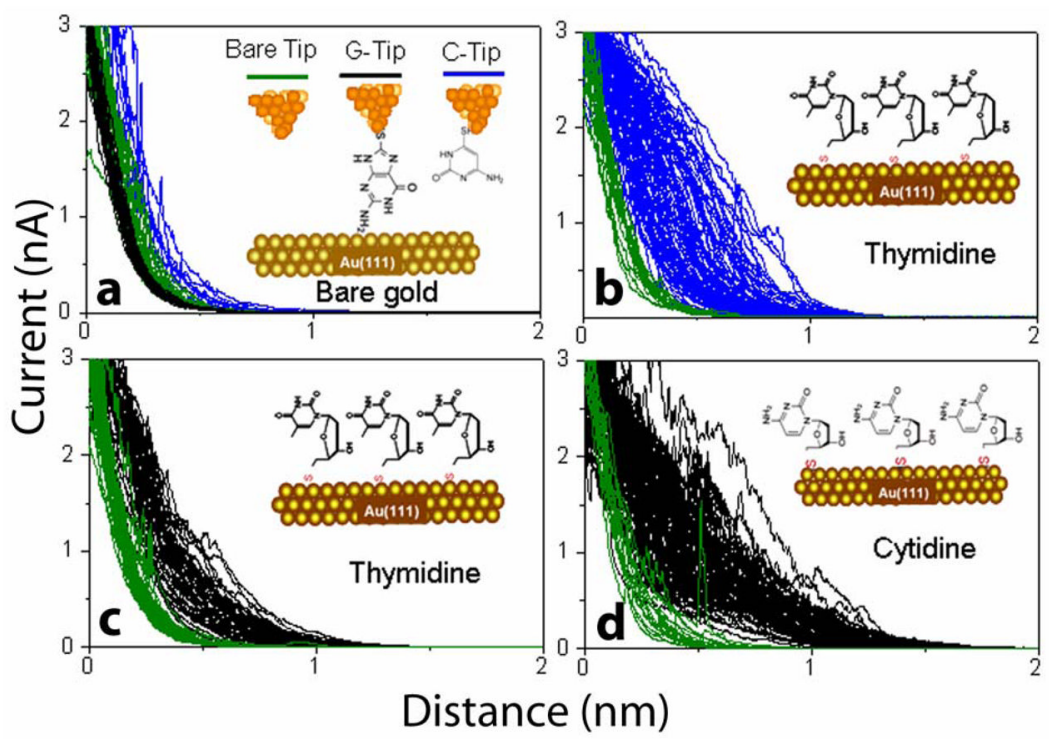

Figure 4.

Raw current vs. distance data taken in water. Color coding and experimental conditions are otherwise as in Figure 1. 

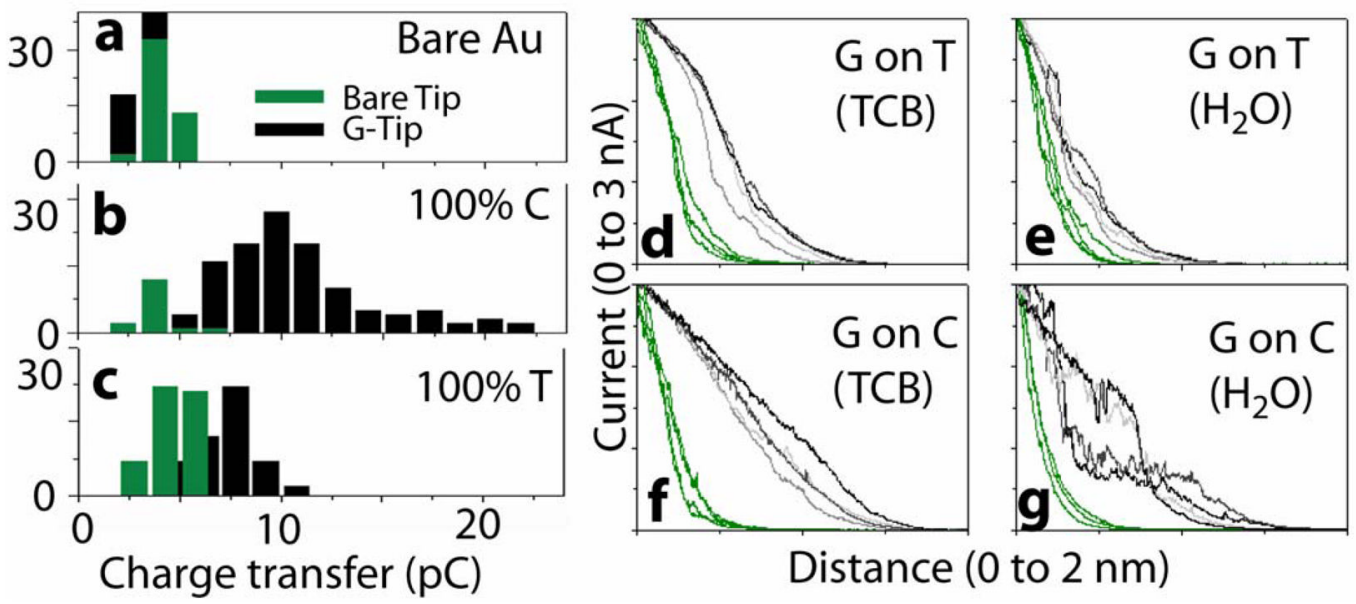

Figure 5.

Analysis of decay curves taken in water. Histograms of the charge transferred in each retraction are shown in (a) (bare Au), (b) (deoxycytidine SAM) and (c) (thymidine SAM). Black bars are for an 8-mercaptoguanine functionalized tip and green bars are for a bare Au tip. Typical i-Z curves in water $(e, g)$ are compared with their counterparts in trichlorobenzene $(d, f)$ showing the complex structure of the data obtained in water (green curves are control data obtained with bare tips). 
Table 1

Average effective decay constants.

\begin{tabular}{|l|l|l|l|}
\hline & \multicolumn{3}{|c|}{ Decav constant $\left(\AA^{-1}\right)$} \\
\hline & Bare & Thvmidine SAM & deoxvcvtidine SAM \\
\hline Bare tip & $0.7 \pm 0.2$ & $0.44 \pm 0.07$ & $0.66 \pm 0.02$ \\
\hline Thio-phenol tip & $0.59 \pm 0.04$ & $0.50 \pm 0.05$ & $0.61 \pm 0.04$ \\
\hline G-tip & $0.68 \pm 0.13$ & $\beta_{1}=0.124 \pm 0.05^{*}$ & $\beta_{1}=0.105 \pm 0.04$ \\
& & $\beta_{2}=0.58 \pm 0.19$ & $\beta_{2}=0.58 \pm 0.27$ \\
\hline & & &
\end{tabular}

* excludes 35\% 'non-bonded' curves (supporting information) 